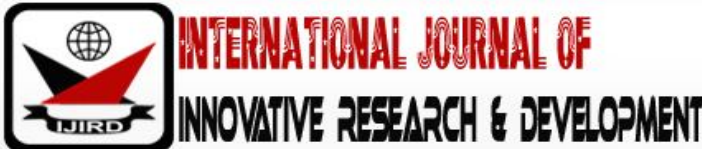

ISSN 2278 - 0211 (Online)

\section{Analysis of SME Micro-Blog Marketing}

\author{
Xu Meng \\ Lecturer, Shangqiu Normal University, Shangqiu, China
}

\begin{abstract}
:
Under the guidance of government related policies, small and medium enterprises in China take the express of the economic development of China's reform and opening up, rely on the regional advantages of the enterprises in the regional economy, and gradually become an important force in the national economy. At present, small and medium enterprises in China are playing an important role in promoting social and economic development, expanding social employment, improving people's livelihood, maintaining social stability and promoting technological innovation. However, due to the late start, low starting point, small scale, small scale, low level of science and technology, inadequate management experience, backward management concept, easy to be crowded with large enterprises and other factors, the development of small and mediumsized enterprises is difficult, especially in the late 90s period of China's economic transition, and the domestic market has been sold from short supply. The square market has gradually shifted to a buyer's market with an oversupply. In the new market environment, how to effectively carry out marketing activities has long been placed in front of small and mediumsized enterprises. Micro-blog marketing is due to the rise and growing popularity of micro-blog. Since 2010, micro-blog has developed rapidly in China. It has become an important social platform after blogs and social networking sites. It has not only built an unprecedented platform for people to communicate faster and convenient, but also created more than now in many ways. There is a media marketing platform. The emergence and development of micro-blog has prompted a brand new way of new media marketing --- micro-blog marketing.
\end{abstract}

Keywords: Small and medium enterprises, marketing activities, micro-blog marketing

\section{Overview}

\subsection{Weibo}

Weibo, the abbreviation of Micro-Blog, is a user-relationship-based information sharing, dissemination, and acquisition platform. Users can access Weibo through WEB, WAP, and various channels, update information with about 140 words and realize timely sharing. The contents of Weibo are usually open. They can publish their own web micro-blogs and can also read other people's micro-blogs. Therefore, they can be understood as the sharing of personal thoughts, ideas, knowledge and so forth on the Internet. There are three elements of Weibo. They are continuous updating of content, reordering of time and diversification of styles.

The earliest and most famous Weibo was the US's Twitter. According to relevant public data, as of January 2010, the product had 75 million registered users worldwide. In August 2009, Sina Website, China's largest portal, launched the "Sina Weibo" closed beta, becoming the first website to provide Weibo services in the portal. Weibo has officially entered the mainstream of China's online population. According to Sina's official statistics, in March 2013, Sina's registered users were 334 million and active users were 30.16 million. As of March 2013, registered users reached 536.5 million and active users reached 60.2 million. The registered users and active users have doubled each year. Tencent Weibo users also reached 507 million in the third quarter of 2012.

\subsection{Micro-Blog Marketing}

With the growing popularity of Weibo among Internet users, various web-based hot words born in Weibo have also become popular online. And the Weibo effect is gradually forming. Micro-blogging is not limited to the user's emotions, information sharing, and interaction. Weibo gradually evolved a new marketing model - micro-blog marketing. 
Weibo Marketing uses Weibo as a marketing platform. Each audience (fan) is a potential marketing target. Enterprises use their own micro-blogs to disseminate corporate information and product information to users and establish a good corporate and product image. Updating content every day to communicate with everyone, or publishing topics of interest to achieve the purpose of marketing is the new way of launched micro-blog marketing. The marketing approach focuses on value transmission, content interaction, system layout, and accurate positioning. The growing popularity of Weibo also makes its marketing effect particularly significant. The scope of micro-blog marketing includes certification, effective fans, topics, famous blogs, open platforms, and overall operations. Of course, micro-blog marketing also has its shortcomings, like insufficient number of effective fans, excessive updates to micro-blog content, excessive demands on writing quality and so forth.

Enterprise micro-blog marketing is a new social marketing model, focusing on the transmission and interaction of information, and is a kind of integration of social resources. Through the rapid dissemination, sharing, interaction, and feedback of micro-blog information, product promotion, crisis public relations, market research, relationship management, and brand communication can be realized. The current U.S. President Barack Obama has used Weibo as a campaign tool in the 2008 presidential election. Voters can participate in the interaction with Obama's campaign team on Weibo, and they can keep abreast of the latest movements and their schedule of Obama's election. Weibo marketing and WeChat marketing have become the most popular marketing methods, especially for those SMEs.

\subsection{Current Status of Micro-Blog Marketing}

The group of Weibo user is generally large group of Internet users in China. Although this group only accounts for about $10 \%$ of Internet users in China, this is indeed a group with relatively high Internet purchasing power. They are particularly sensitive to new things. The post-80s are dominated, and the post-90s also account for a large part of them. They are the main force for future consumption. In addition, in recent years, China's SMEs have continued to develop with the benefits of reform and opening up. SMEs have emerged throughout the country like mushrooms after the rain. However, these small and medium-sized enterprises are often at a disadvantage in the competition with their counterparts in the industry due to their low capital, weak strength, and low advertising investment. They have to survive in the slits of several oligarchs. Therefore, small and medium-sized enterprises especially need Weibo's low-threshold, extensive coverage and threedimensional social marketing platform to serve them. This will also lay the foundation for the continued development of micro-blog marketing in the future. Since December 2012, Sina Weibo has launched a corporate service provider platform to provide businesses with some help in marketing on Weibo.

However, various disadvantages such as the rapid development of micro-blogging marketing have also emerged. For example, if corporate micro-blogging marketing is improperly used, public relations crisis may occur; In addition, due to the lack of professional micro-blogging marketing team or micro-blogging marketing talents, it is easy to get trapped in various misunderstandings during micro-blogging Marketing, like unclear goal, insufficient coherence, lack of interactive interaction with fans, blindly rewarding or setting gifts to stimulate the growth of fans, unreasonable micro-blogging design that cannot arouse the interest of users. And even worse, some companies only maintain the corporate micro-blogs as windows of information transmission. All this have severely constrained the development of SMEs in China.

\section{Analysis on the Characteristics of Micro-Blogging Marketing}

\subsection{Features of Micro-Blogging Marketing}

The first one is low threshold. Information with 140 words does not need to make too many ideas, which is far easier than blog publishing.

The second one is about its three-dimensional feature. Micro-blogging marketing can use advanced multimedia means to describe products from text, pictures, videos and other forms of presentation.

The third one is fast speed. One of the most significant features of micro-blog is its fast speed of dissemination. A highly concerned micro-blog can reach all corners covered with micro-blog after being published on the Internet and its associated mobile WAP platform within a short time, and thereby achieving the result that the number of the witnesses is the largest in the shortest time.

The fourth one is very convenience. Micro-blogging Marketing is superior to any traditional advertising industry. The main body of published information does not need frequent administrative approval, and thus saving a lot of time and cost. The fifth one is extensiveness. The information is spread like virus in the form of followers' attention, with a wide range of influence. At the same time, the celebrity effect can make the amount of information spread into geometric magnification.

The sixth one is cross-border. Compared with traditional interactive marketing, micro-blog's interactive form can break the geographical and number restrictions, and global audiences may become participants in interactive marketing. More importantly, like-minded people from different regions can communicate in real time and have a deeper understanding. Brand awareness will increase in communication and attention.

The seventh one is low cost. Compared with traditional marketing methods, it does not require high costs. Using 
micro-blog to publish relevant product information almost cost nothing.

The eighth one is high pertinence. The users who focus on the company or product micro-blog are generally the company's customers or potential customers, and the company can accurately place advertisements.

The ninth one is high interaction. Micro-blog marketing is a two-way interactive exchange. Merchants can collect user's feedback in a timely manner, provide necessary basis for product improvement and production and increase customer loyalty.

\subsection{Weaknesses of Micro-Blog Marketing}

First, popularity is the basis of micro-blog marketing. If there aren't a large number of fans for reprinting, the effectiveness of micro-blog marketing will be greatly reduced. It is difficult for small and medium-sized enterprises to accumulate a large number of fans in a short period of time due to insufficient visibility. And they usually cannot find clues in micro-blogging marketing.

Second, micro-blog content is updated quickly. If the fans do not see it in time, this information may be submerged in the vastness of the information. Therefore, no communication effect can be achieved in such a situation.

Third, the content of the dissemination is limited because a micro-blog generally requires about 140 words. It is impossible for people to deliver the information that they want to convey. People cannot grasp the main point of the micro-blog and the number of reprints may not be more than that of blogs. If content of a micro-blog lacks reasonable processing and packaging, the effect of the communication may be worse unless it is reproduced by an influential Internet star or celebrity.

Fourth, the cycle is long and the effect comes slow. When a small unknown and medium-sized enterprise carries out micro-blog marketing, it will often encounter that although the content released is of high quality and valuable to users, it is usually not good in information dissemination due to low attention of users. If companies want to use micro-blog for marketing, they must constantly maintain micro-blog. They must pay attention to the number of micro-blog, focus on quality and communicate with fans in a timely manner to attract more attention.

Fifth, the reliability of micro-blog marketing has been questioned. In the minds of consumers, the reliability of online media is often not comparable to traditional

print media. Integrity in micro-blog marketing is the ultimate thing. Just like decentralized bitcoin, bitcoin will cease to exist due to the lack of trust.

Sixth, the demand for writing quality is too high. The advertised product information should be accepted by the fans. If too much information about the company's products is used in micro-blog, it will have the opposite effect. The defenders of corporate micro-blogs generally need professional training or a micro-blog marketing team.

\section{Example of Case -BST Micro-Blog Marketing}

Nanyang International, Manufacturer of BST, is a private joint-stock enterprise. It is a professional cosmetic enterprise integrating brand management and product processing. This company invested 90 million yuan in 2008 to build a large-scale cosmetics production base in Happiness Industrial Park of Nantong Gangzha District. The base is next to the Ningtong Expressway, 25-minute-journey away from Nantong Airport and one-hour-travel away from Shanghai. The traffic is very convenient.

\subsection{Purpose of the Brand}

"Be beautiful in a more natural and healthier way" is the purpose of BST. Hundreds of years of experience in natural herbal medicine selection, combined with modern biochemical extraction technology, has achieved the quality of today's BST, bringing good news for Asian women to maintain beauty. The main representative products of BST are Condensate Moisturizing Face Mask, Active Whitening BB Cream, Skin Care Whitening Moisturizing Toner, Sleeping Mask, Whitening and Moisturizing Cream, Whitening Facial Cleanser and so forth. The company also signed the most popular stars Liu Shishi and Ying Er as their endorsements, making BST quickly known by local consumers.

\subsection{Growth of BST}

On July 31, 2011, "Poetry World" was held to promote BST's related products through the popularity and activities of spokesperson Liu Shishi. The BST again joined hands with Liu Shishi in the "Beauty Poetry Creation Competition" held on August 1st, 2011. The event was not only promoted on major websites, but also broadcasts on BST's officialWeibo and Liu Shishi Bar's micro-blog, with follow-up reports. On August 6th, 2011, colorful pictures were organized to fight together. Similar to the previous events, the prizes were related products of BST. Through a series of activities, consumers' awareness of BST brand was 
deepened, and thus broadening the audience of BST.

BST also registered micro-blog accounts such as BST Miniscule, BST's Crossing a Century of Beauty, and "BST's Little Coupons in Life", which made BST involved at all levels. The following is a tweet about BST.

BST wishes everyone a happy new year. Now friends who have become the fans of @BST-Official Weibo, and reprinted this micro-blog and @ more than five friends will have the chance to get BST souvenirs, a step-by-step DVD box, the BST print ads autograph fan of Shishi. Time: From December 31st to January 12:00am. The winners will be announced on January 3rd! $@$ Liu Shishi @The poetry dance - the official fans website of Liu Shishi.

@The talent era : \#short novel\# She is a skin-care activist. One day, she walked on the way to work and encountered a skin care product conference. However, it was sold out when she arrived. She cried. She turned to leave after cleaning her eyes when a man shouted towards her direction, "Maertai Losi, what the hell do you want!" She responded with a shock, "I want BST!" @BST-Official Weibo Address: Http:t.cn/ SPmh7d

The third part of Autumn skin care is moisturizing. The BST Condensed Moisturizing Cream endorsed by @Liu Shishi can soothe and repair the skin, condition skin tone, replenish skin with a lot of moisture, smooth fine lines and make skin be white and smooth again; It contains rich herbal essence like cactus extraction materials and so forth. It is quite skin-friendly, fresh and non-greasy and provides long-lasting moisture and nutrients to the skin, leaving the skin staying translucent and moisturizing for a whole day. @ Liu Shengnan 1989

\subsection{Current Status of BST Marketing}

Up to now, BST promotion activities have been held for dozens of times. The number of Fans related to BST has developed from hundreds of people at the beginning to millions at present. And most of the users who participated in the activities or users of the products have become loyal fans of BST. These fans also act as active activists, the diffuser of good reputation, or potential users of the BST... The stickiness of these fans is very solid and they are real users or potential users.

\subsection{PRAC Marketing of BST Micro-Blog}

First, look for and pay attention to those users or fans who have discussed BST and join their dialogues so that fans feel highly valued and recognized, inspiring discussion and spreading enthusiasm.

Second, hold a series of BST activities, continue to stabilize the fans' enthusiasm and allow fans to continuously expose and honor the event.

Third, organize fan conversations or suggestions on the company's micro-blog, make stories with interaction with fans spread widely to inspire fans and make fans feel recognized and respected to fully embody humanity.

Fourth, establish an active fan group, or discussion group, allow fans to interact and communicate and then collect their suggestions and opinions on the product.

Fifth, give fans a variety of rewards, such as red envelopes, free trial equipment, advertising participation and other rewards.

\subsection{SWOT Analysis on the Micro-Blog Marketing of BST}

\subsubsection{Analysis of Advantages}

First, the publishing threshold is low. For a micro-blog, even a photo or a signature can become an advertisement and serve as a marketing service for enterprises.

Second, it has convenient channels, fast information dissemination and wide range. Micro-blog marketing uses the most convenient way of marketing - online marketing. BST's micro-blog marketing not only enables faster information dissemination but also enables consumers to feedback on the effect of the product to the network, making the product more realistic. Most people are using mobile phones to pay attention to Weibo, which allows product information to spread faster and wider and to form a viral marketing model that is more convenient, extensive, and practical.

Third, it is equipped with Affinity-based communication and strong interaction between companies and users. BST has captured one of Weibo's characteristics, that is interaction and equality. Micro-blog marketing allows fans to have the same opportunities for dialogue, communication and experience. They no longer look up to the brand but talk about brand products with an equal attitude. It is up to the fans to decide whether to comment, post, or participate.

Fourth, it has a low cost of information: BST is not well-known, and the number of visits is also relatively small. Just using weibo as a link to a website increases the visibility of web searches on major search engines. This cost is extremely low. 


\subsubsection{Analysis of Disadvantages}

The first one is the proliferation of low thresholds. Under the circumstances that domestic micro-blog marketing is not mature enough, BST micro-blog is just one of countless cosmetics brands. Faced with such a large number of competitors, its micro-blogmarketingis beingimitated by various companies. The proliferation of gray marketing overdrew the trust of Internet users, resulting in a substantial increase in the number of fans but little improvement in sales.

The second one is that it can be easily used by its competitors. If you do not do well in the process of information transmission such as internal employees' accidental leakage of information, the open micro-blog fans and so forth can provide the convenience for the opponent companies to obtain the core information.

The third one is that it is easy to generate public relations crisis. Due to the timeliness of micro-blog content and the extremely fast propagation speed, the reaction time of micro-blogmarketing has been greatly shortened, which greatly increased the production factor of network public relations issues. Therefore, how to give warning before the public relations crisis occur and how to deal with the public relations crisis are all of great importance.

\subsubsection{Analysis of Opportunities}

The first one is the decline in the attractiveness of traditional marketing methods. With the convenience of network technology for consumers, traditional marketers have lost a lot of market share, and the management of enterprises have to be positioned along with changes in the market. It will also rely on the network instead of traditional materials. The decline in the attractiveness of traditional marketing methods also provides BST with a good opportunity for the growth of micro-blog marketing.

The second one is the combination of the development of E-commerce with micro-blogging. The emergence of $\mathrm{E}$ commerce has changed people's understanding of the market. And the E-commerce approach has played down the status of the tangible market in commodity exchange. With the improvement of various $\mathrm{B} 2 \mathrm{~B}, \mathrm{~B} 2 \mathrm{C}$ and $\mathrm{C} 2 \mathrm{C}$ electronic platforms, it has gradually become one of the mainstream sales platforms. Therapid development of Ecommercealso provides opportunities for thegrowthand maturity of micro-blog marketing.

\subsubsection{Analysis of Threats}

First, sensible sales cannot serve as the sole responsibility of independent marketing. Although micro-blog marketing can be market segmented based on the characteristics of consumers' age, hobbies and so forth, a sales plan can be drawn up. However, micro-blog marketing still relies on emotions to agglomerate consumers. Nevertheless, in the sales process, perceptual sales are far from rational sales and focus on product performance. Micro-blog marketing stimulates the emotional closeness of consumers through various channels of communication, making it depend on this to some extent. However, pure emotional sales cannot bear the burden of sales independently, and the lack of rational sales will also put the company into crisis.

The second one is about the threats from various SNS websites. The number of users of various types of SNS websites now exceeds the number of micro-blog users. This is because SNS is more real than micro-blog. It often uses real-name registrations such as Renren website and Kaixin websites. And SNS websites have more functions than micro-blog. Both music and games are better than that of micro-blog. Threats from websites have forced the major micro-blog platforms to continuously improve themselves.

\section{The Strategies of SMEMicro-Blog Marketing}

\subsection{Pay Attention to the Celebrity Effect in Micro-Blog Marketing}

The celebrities in micro-blog marketing include online celebrities, pop stars, industry leaders, Internet insiders and civilian stars. Celebrities generally have strong attractiveness and influence. The celebrity effect is like a branding effect. It can drive people. Its effect is as powerful as that of chasing a star. If SMEs can reasonably use the celebrity effect in micro-blog marketing, then the micro-blog marketing will be spread in a faster way, wider range and last for a longer time.

Public figures generally have a high reputation, with a large number of fans. The use of celebrities for micro-blog marketing can significantly increase the company's or corporate products' image and popularity. Celebrity endorsements are more persuasive and appealing. When users see or hear the celebrity, they may think of the product of the company or the enterprise. 


\subsection{Rational Use of Micro-Blog for Integrated Marketing}

Micro-blog marketing is just an innovative method of online marketing. However, the active users of Weibo and the coverage of the crowd are limited. It is sometimes difficult to achieve good communication results by using pure micro-blog marketing. At the same time, there are many online media channels of marketing that can be used outside of Weibo, such as the People's Network, Sina, Tencent, Netease, Sohu, Hexun and other public relations resources; There are platforms of the "We Media" type like NetEase Cloud Reading, Sohu News All-Media Platform, WeChat Public Platform, the Public Platform of Laiwang and the e-trust public platform. There are also platforms of the social networking type, such as Renren Website, Douban group, Kaixin Website, Baidu Postbar, QQ, WeChat, all kinds of social BBS and so forth. There are also platforms belonging to the science and technology type, like Baidu Knows, Zhihu and so on. Small and medium-sized enterprises can choose the suitable network marketing channels for integrated marketing on Weibo based on their own conditions and target customer groups.

However, sometimes these online channels do not fully cover most of the customer groups of SMEs. For example, the products of some small and medium-sized enterprises are mainly aimed at middle and old age groups. But many middle-aged and old people do not have access to the Internet and most of the time, their children are not around. Therefore, SMEs must pay attention not only to online but also offline when they conduct integrated marketing and carry out online and offline activities simultaneously

\subsection{Pay Attention to Team Building of Micro-Blog Marketing}

The successful micro-blog marketing is not because of the work of a single person but a professional micro-blog marketing team behind. Micro-blog marketing requires teamwork and a certain amount of expertise. Weibo marketing is systematic and continuous information dissemination. The general process of a successful micro-blog marketing case includes the customization of micro-blog, the operation of micro-blog, the promotion of micro-blog, the planning of micro-blog events, the speculation of micro-blog, the launch of micro-blog, the public relations of micro-blog, the monitoring of information, the report of micro-blog, evaluation, micro-blog APP applications and so on. Every step requires people to be responsible for execution. Any problem in each part of the process can lead to the failure of micro-blog marketing activities and even produce a serious public relations crisis. Therefore, SMEs need to pay attention to the training of micro-blog marketing talents and the construction of micro-blog team when conducting micro-blog marketing. They regularly provide training on micro-blog marketing to employees and continuously deal with the drawbacks and problems in micro-blog marketing. At the same time, it is necessary to draw lessons from previous failed micro-blog marketing cases and take them as a warning; Sum up experience from successful cases, draws on the essence and destroy its dross.

\subsection{Seek Truth from Facts and Start from Reality}

Although Micro-Blog marketing and Wechat markets are the most popular marketing methods at the moment. However, the application of these two marketing methods should be based on actual needs. Do not blindly follow trend. For example, for some small-scale catering companies, the scope of services is generally limited to a fixed area and the customer base is generally the surrounding residents. Such companies are not suitable for micro-blog marketing. It is still difficult for them to achieve the desired results even if the micro-blog marketing is conducted. It is purely a waste of resources. Such SMEs should focus on improving product quality and service attitude. Due to the lack of professional micro-blog marketing talents or teams, some small and medium-sized enterprises are prone to be trapped into various misunderstandings in micro-blog marketing, some of which even lead to public relations crisis. Before carrying out micro-blog marketing, they should learn more basic knowledge of network marketing and some courses of micro-blog marketing.

Some small and medium-sized enterprises even think that there will be benefits to the company as long as they set up Weibo. After spending a lot of resources and manpower, they find that micro-blog marketing is not as simple as they think. They then began to learn about WeChat marketing after seeing that there are lots of companies conducting WeChat marketing but they finally ended up with nothing. Therefore, small and medium-sized enterprises are advised to choose the appropriate form of network marketing according to their own needs. Remember not to follow others blindly.

\subsection{Adhere to the 4I Principle of Micro-Blog Marketing}

The 4I principle of micro-blog marketing refers to the principle of interaction, interest, interesting and individuality.

\subsubsection{Principle of Interaction}

One of the most important aspects of micro-blog marketing is interaction. In the process of interaction, you can become closer with your fans and make them be loyal to you. If you do not interact effectively with your fans, some fans will leave 
because of your arrogance. There are times when you carry out some prized-and-forward commentary activities. The only thing that attracts fans is the prize. After the event, those fans may choose to leave. You can respond seriously to the comments and private letters of everyone fans, exchange your actions for the recognition of fans, and make them automatically integrate into the interactive circle in the future days. Because most of the people in China have crowd psychology, the popularity of corporate micro-blogs will gradually increase as the interaction increases and the audience will become more and more extensive. When encountering negative information, do not make blind announcements. Instead, contact the parties in a timely manner to conduct effective communication and carry out appropriate crisis management.

\subsubsection{Principle of Interest}

Micro-blog marketing is a mutually beneficial and win-win marketing concept. Corporate micro-blog is not only a transmission window for corporate information, values and business concepts, but also a carrier of corporate value and corporate image. In addition, there are thousands of micro-blog messages every day. Visitors generally have interests in valuable information. Enterprises can only get benefit by meeting the user's requirements. Therefore, when companies conduct micro-blog marketing, they notonly need to promotethemselves butalso requirestodelivervaluableinformation to customers. For example,Delloften publishessome discountinformationand spikeinformation through micro-blog.

\subsubsection{Principle of Being Interesting}

Micro-blog cannot be apart from this entertainment era. Whether it is on Twitter abroad, or domestic Sina Weibo, Sohu Weibo, Tencent Weibo, the most eye-catching is either a major news event or a humorous paragraph for all ages, orafunny picture, or a funny video. Weibo users do not liketouseofficial words and micro-blogs with less fun. Without followers' attention, reprint and comment, the micro-blog marketing of enterprises will fail. There is a sayingcirculating on the Internet that gossip is the passport to hot and Steamed Bread is the epitaph of The Promise. The popularity of Sister Lotus's big-sized body is showing the importance of entertainment. Therefore, small and medium-sized enterprises should pay attention to the fun of micro-blog when conducting micro-blog marketing.

\subsubsection{Principle of Individuality}

There are thousands of companies that use micro-blog marketing and the competition for micro-blog marketing is also very fierce. Those marketing methods that do not have their own characteristics and follow others blindly can easily cause people to become aesthetically fatigued. Only innovative and attractive accounts can stand out from the crowd of corporate micro-blogs. Just like people in real life, personality is an important basis for distinguishing people from people. And it is also the basis for people to exist in this society. Personal charm is where people's core values lie. The charm of Western presidential candidates is often one of the key factors in the success or failure of election campaigns. Customers are more likely to be captured because of individuality. It is so accurate due to individuality; It is so attractive because of individuality. The individuality of the company's micro-blogging marketing represents the corporate image in the network. Therefore, the company should adhere to the principle of individuality of micro-blog in micro-blog marketing.

To sum up, Weibo has a high speed of dissemination, a wide range of concerns and a low cost. New marketing methods for actively managing target customers are particularly welcomed and appreciated by SMEs. As a new enterprise marketing tool, its advantages have been presented. It has broad application prospects and commercial value. Through this kind of humanized exchange, companies can get a higher degree of attention, and then enhance the company's brand value. Therefore, SMEs must use innovative marketing thinking to fully use micro-blog marketing according to their own product characteristics, create value for corporate marketing, and make themselves invincible in the fierce competition in the market.

\section{References}

i. Zou Wei. Blog Marketing - New Tool for Internet Marketing [J] E-commerce World. 2006

ii. Dong Keju. Introduction to Human Resource Management. Second Edition. China Renmin University Press.

iii. Chen Yinghui, Hu Haibo. Analysis on the Role of Blogs in Online Marketing [J] Modernization of shopping malls, 2006

iv. Fang Jinbiao. Analysis of how SMEs Use Micro-Blog for Online Marketing [J] Wuxi Vocational and Technical College 2009

v. Wang Wei. About the Rational Use of Network Marketing of Enterprises [J] Jiangxi University of Finance and Economics 2011

vi. Wang Dan. Analysis of the Status and Development Trend of Enterprise Blog Marketing [J]. Journal of Wuhan Institute of Business Services, 2007

vii. Zhang Dayi. The Distribution Channels of Enterprises in the E-commerce Environment [J] Contemporary Economy, 2010 
viii. Zhou Sanduo. Management [M] Higher Education Press, 2010

ix. Hu Zhaohui. Management [M] Chemical Industry Press, 2010

x. Liu Hongju. Li Yushi. Tian Weilin. Research on the Marketing Model of Micro-Blog Brand [J] Thesis Net, 2012 